\title{
Epífora congênita nos pacientes com síndrome de Down
}

\author{
Congenital epiphora in patients with Down syndrome
}

\author{
Christiane Carvalho Salvio ${ }^{1}$ \\ Wilson Takashi Hida ${ }^{2}$ \\ José Vital Filho ${ }^{3}$ \\ Marcos Cunha $^{4}$ \\ Joelice dos Santos Araújo ${ }^{5}$
}

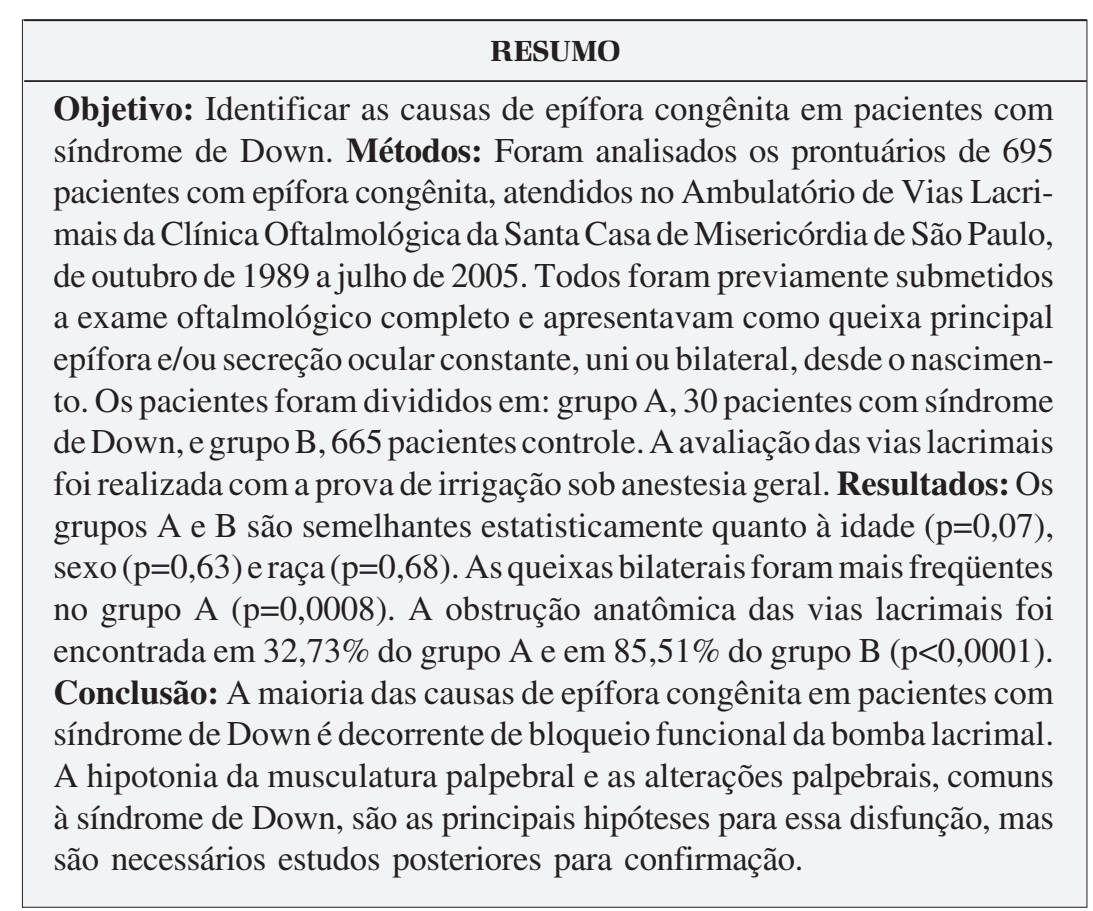

Descritores: Doenças do aparelho lacrimal/congênito; Obstrução dos ductos lacrimais/ etiologia; Síndrome de Down/complications; Oftalmopatias

Trabalho realizado no Departamento de Oftalmologia da Santa Casa de Misericórdia de São Paulo.

${ }^{1}$ Residente do 3ำ ano do Departamento de Oftalmologia da Santa Casa de Misericórdia de São Paulo - São Paulo (SP) - Brasil.

${ }^{2}$ Residente do $3^{\circ}$ ano do Departamento de Oftalmologia da Santa Casa de Misericórdia de São Paulo - São Paulo (SP) - Brasil.

Chefe do Setor de Órbita do Departamento de Oftalmologia da Faculdade de Ciências Médicas da Santa Casa de Misericórdia de São Paulo - São Paulo (SP) - Brasil.

${ }^{4}$ Chefe do Setor de Plástica-Ocular do Departamento de Oftalmologia da Faculdade de Ciências Médicas da Santa Casa de Misericórdia de São Paulo - São Paulo (SP) - Brasil.

${ }_{5}$ Chefe do Setor de Vias Lacrimais do Departamento de Oftalmologia da Faculdade de Ciências Médicas da Santa Casa de Misericórdia de São Paulo - São Paulo (SP) - Brasil.

Endereço para correspondência: Wilson Takashi Hida. Rua Afonso de Freitas, 488 - Apto. 61 - São Paulo (SP) CEP 04006-052

E-mail: witaks@yahoo.com.br

Recebido para publicação em 05.01.2006

Última versão revisada em 24.07.2006

Aprovação em 30.08.2006

Nota Editorial: Depois de concluída a análise do artigo sob sigilo editorial e com a anuência do Dra. Ana Estela Besteti Pires Ponce Sant'Anna sobre a divulgação de seu nome como revisora, agradecemos sua participação neste processo.

\section{INTRODUÇÃO}

A síndrome de Down constitui a alteração cromossômica mais freqüente do ser humano ${ }^{(1)}$. Essa denominação é resultado da descrição de Langdon Down*, médico inglês que, pela primeira vez, descreveu, em 1866, as características clínicas de uma criança com essa síndrome. Três tipos de anomalias cromossômicas podem ser encontradas: trissomia livre do cromossomo 21 , translocação e mosaicismo ${ }^{(2)}$.

O diagnóstico da síndrome de Down é feito através da cariotipagem cromossômica, que pode ser realizada, durante a gestação, por análise citogenética das vilosidades coriônicas ou células do líquido amniótico ${ }^{(2)}$.

Embora a base cromossômica da síndrome de Down seja clara, o motivo da anomalia cromossômica ainda é pouco compreendido. O aumento da incidência, com a idade materna avançada, foi primeiro enfatizado por Shuttleworth $(1909)^{* *}$ e confirmado em vários estudos subseqüentes. Sugeriu-se

\footnotetext{
* Down JLH. Observations on an ethnic classification of idiots. Clinical Lectures and reports by Medical an Surgical Staff of the London Hospital Resports. 1866;3:259-62. apud ${ }^{(2)}$.

**Shuttleworth GE. Mongolian imbecility. Br Med J. 1909;3:661-5.apud (2).
} 
que, quanto mais antigo for o ovócito, maior será a chance de que os cromossomos não se disjuntem corretamente ${ }^{(2-3)}$.

Pais de criança com síndrome de Down têm risco aumentado de ter outro filho com essa síndrome em gestações futuras, principalmente nos casos de translocação; por essa razão o aconselhamento genético é importante ${ }^{(2,4)}$.

No Brasil, a incidência é cerca de 1,13:1.000 nascidos vivos ${ }^{(5)}$.

A síndrome de Down em geral pode ser diagnosticada ao nascimento ou logo após, por suas características dismórficas, que variam entre os pacientes, mas sempre produzem um fenótipo característico. A hipotonia muscular pode ser a primeira anomalia notada nos neonatos ${ }^{(2)}$. Hall, em 1966, encontrou hipotonia em $80 \%$ dos recém-nascidos com síndrome de Down. Os pacientes têm baixa estatura, braquicefalia, pescoço curto, com pele frouxa na nuca ${ }^{(6)}$. A ponte nasal é achatada, as orelhas têm implantação baixa, com aspecto dobrado característico, a boca permanece aberta, mostrando macroglossia e cheia de sulcos. As pregas epicantais e as rimas palpebrais oblíquas deram origem ao termo "mongolismo", que hoje é considerado impróprio e não deve ser usado. As mãos são curtas e largas e os pés mostram um grande espaço entre o primeiro e segundo artelhos ${ }^{(2)}$.

O quociente de inteligência em geral é de 30 a 60. Felizmente, o desempenho social desses pacientes costuma ser superior ao que seria de se esperar de acordo com a idade mental; são geralmente lactentes dóceis e crianças alegres e responsivas ${ }^{(2,4)}$.

As malformações cardíacas constituem a principal causa de mortalidade precoce. As infecções das vias respiratórias baixas são por vezes sério problema e há um aumento de 15 vezes no risco de leucemia ${ }^{(1-2,4)}$.

As alterações oculares mais citadas, como características da síndrome, em ordem de incidência, são: rimas palpebrais oblíquas, epicanto, ametropias, anomalias de íris (manchas de Brushfield), estrabismo, blefarite, obstrução das vias lacrimais, alterações retínicas, ambliopia, nistagmo e catarata ${ }^{(3,5,7-8)}$.

A epífora é a queixa mais freqüente desses pacientes. É importante a diferenciação para correto diagnóstico e tratamento.

O lacrimejamento significa hiperprodução lacrimal. A lágrima é secretada em excesso, não sendo possível a drenagem total. Pode ocorrer em doenças da superfície ocular, pálpebras, inflamações da úvea e glaucoma congênito ${ }^{(9)}$.

A epífora é definida como o transbordamento da lágrima pela rima palpebral decorrente de seu escoamento insuficiente. É o sinal mais freqüente das afecções do sistema de drenagem lacrimal e pode ocorrer em decorrência de fatores como ectrópio palpebral, bloqueio funcional da bomba lacrimal e obstrução anatômica parcial ou total do fluxo lacrimal, em qualquer nível do seu trajeto $^{(9)}$.

A blefarite é encontrada em cerca de $30 \%$ dos pacientes com síndrome de Down ${ }^{(5)}$. Shapiro, France, observaram essa alteração em $46 \%$ dos pacientes do seu estudo ${ }^{(10)}$. Catalano acredita que a alta frequência de blefarite, na síndrome, esteja relacionada com a menor capacidade de combater infecções que essas crianças possuem ${ }^{(11)}$.

O funcionamento adequado do sistema lacrimal depende da função normal do músculo orbicular, do posicionamento adequado dos pontos e canalículos lacrimais e da presença de vias lacrimais pérvias ${ }^{(9,12)}$.

Os achados oculares externos, como a rima palpebral oblíqua, o epicanto e o estreitamento da distância interpupilar são as características mais fortemente associadas à síndrome de Down $^{(3)}$. Acreditamos que essas alterações modifiquem o posicionamento dos pontos e canalículos lacrimais e prejudiquem a ação do músculo orbicular, comprometendo o funcionamento da bomba lacrimal.

A rima palpebral com inclinação oblíqua foi a característica ocular mais freqüentemente encontrada nos pacientes com síndrome de Down, em vários estudos ${ }^{(3,5,7,10,13)}$. A medida da inclinação da rima palpebral é realizada geralmente pelo método descrito por Solomon et $\mathrm{al}^{(14)}$. A margem superior de uma régua transparente é posicionada na base do nariz, nos cantos mediais de ambos os olhos, e é medido o deslocamento vertical em relação aos cantos laterais.

A prevalência de obstrução das vias lacrimais, em pacientes com síndrome de Down, varia na literatura. Alguns autores em 1986, encontraram prevalência de 30\% de alterações nas vias lacrimais $^{(5)}$, enquanto Catalano, em 1990, observaram que a obstrução do ducto lacrimonasal é um achado raro e provavelmente não relacionado à síndrome ${ }^{(11)}$.

Alguns autores demonstraram que a obstrução anatômica das vias lacrimais em pacientes com síndrome de Down, ocorre entre o saco lacrimal e o ducto lacrimonasal ${ }^{(1)}$.

O crescente afluxo de pacientes com síndrome de Down em nosso ambulatório nos últimos anos e a dificuldade de liberação clínica, decorrente das alterações sistêmicas, para a propedêutica das vias lacrimais sob anestesia geral, motivou-nos a realizar este estudo retrospectivo, para tentar identificar a real incidência de obstrução anatômica das vias lacrimais e a necessidade do estudo das vias lacrimais nesses pacientes. Não encontramos na literatura trabalhos abordando esse tema.

\section{OBJETIVO}

O objetivo do presente estudo foi identificar as causas de epífora congênita nos pacientes com síndrome de Down, avaliando a incidência de obstrução anatômica das vias lacrimais e bloqueio funcional da bomba lacrimal, assim como comparar essas incidências com a de pacientes controle.

\section{MÉTODOS}

O presente estudo foi aprovado pelo Comitê de Ética em Pesquisa em Seres Humanos da Irmandade da Santa Casa de Misericórdia de São Paulo, com protocolo número 280/05, de 5 de setembro de 2005.

Estudo retrospectivo realizado mediante análise dos prontuários de pacientes com menos de 20 anos e epífora congênita, atendidos no Ambulatório de Vias Lacrimais do Departa- 
mento de Oftalmologia da Santa Casa de São Paulo, de outubro de 1989 a julho de 2005.

Foram excluídos do estudo, pacientes atendidos no ambulatório de Vias Lacrimais que apresentavam tumor, abscesso nas vias lacrimais, doenças da superfície ocular ou pálpebras.

Todos foram previamente submetidos a exame oftalmológico completo e apresentavam como queixa principal epífora e/ou secreção ocular constante, uni ou bilateral, desde o nascimento.

Os pacientes foram separados em dois grupos: grupo A, 30 pacientes com diagnóstico de síndrome de Down e grupo B, 665 pacientes sem a síndrome.

A média de idade e desvio padrão em anos dos pacientes, no momento da realização da prova de irrigação das vias lacrimais, foi de 5,50+/-5,59 (0,75 a 19,83) no grupo A e 3,17 $+/-2,81(0,25$ a 19,50$)$ no grupo B.

A distribuição dos pacientes segundo a faixa etária é mostrada no quadro 1 .

As características da amostra em relação ao sexo, raça e lateralidade das queixas são apresentadas no quadro 2.

Os pacientes dos dois grupos foram submetidos à prova de irrigação das vias lacrimais em ambos os olhos, pelo médico assistente ou estagiário do serviço de Vias Lacrimais, sob anestesia geral inalatória com máscara laríngea.

Após o procedimento anestésico, iniciava-se o exame com a dilatação do ponto lacrimal inferior. Uma sonda vesical 6.0 era introduzida na cavidade nasal ipsilateral. Uma cânula de vias lacrimais de diâmetro $0,7 \mathrm{~mm}$, conectada a uma seringa de

Quadro 1. Distribuição por faixa etária, no momento da realização da prova de irrigação das vias lacrimais, dos pacientes do grupo A e B

$\begin{array}{lcr}\text { Idade } & \text { Grupo A }-\mathbf{n}(\%) & \text { Grupo B }-\mathbf{n ~ ( \% )} \\ 0-5 \text { anos } & 19(63,33) & 526(79,10) \\ 5-10 \text { anos } & 5(16,67) & 112(16,84) \\ 10-15 \text { anos } & 3(10,00) & 21(3,16) \\ 15-20 \text { anos } & 3(10,00) & 6(0,90)\end{array}$

Grupo A: pacientes com síndrome de Down; Grupo B: pacientes controle.

Fonte: Ambulatório de vias lacrimais do Departamento de Oftalmologia da Santa

Casa de São Paulo no período de outubro de 1989 a julho de 2005.

\begin{tabular}{|c|c|c|}
\hline \multicolumn{3}{|c|}{$\begin{array}{l}\text { Quadro 2. Sexo, raça e lateralidade das queixas de epífora e/ou } \\
\text { secreção ocular constante dos pacientes do grupo A e B }\end{array}$} \\
\hline & Grupo A - n (\%) & Grupo B - n (\%) \\
\hline \multicolumn{3}{|l|}{ Sexo } \\
\hline Feminino & $15(50)$ & $291(43,37)$ \\
\hline Masculino & $15(50)$ & $374(56,24)$ \\
\hline \multicolumn{3}{|l|}{ Raça } \\
\hline Branco & $24(80)$ & $498(74,88)$ \\
\hline Não branco & $6(20)$ & $167(25,12)$ \\
\hline \multicolumn{3}{|c|}{ Lateralidade das queixas } \\
\hline Monocular & $5(16,67)$ & $330(49,62)$ \\
\hline Binocular & $25(83,33)$ & $335(50,38)$ \\
\hline \multicolumn{3}{|c|}{$\begin{array}{l}\text { Grupo A: pacientes com síndrome de Down; Grupo B: pacientes controle. } \\
\text { Fonte: Ambulatório de vias lacrimais do Departamento de Oftalmologia da Santa } \\
\text { Casa de São Paulo no período de outubro de } 1989 \text { a julho de } 2005 \text {. }\end{array}$} \\
\hline
\end{tabular}

5,0 cc contendo água destilada e fluoresceína era introduzida no canalículo inferior e a solução injetada. A fluoresceína, dilúida em água destilada, era usada como corante, para facilitar a identificação da passagem da solução pela sonda nasal.

A passagem de líquido corado pela sonda nasal significa vias lacrimais pérvias e a epífora é decorrente da função inadequada da bomba lacrimal. Se ocorrer distensão do saco lacrimal ou refluxo da solução, com ou sem secreção, pelo canalículo superior e/ou inferior conclui-se que existe obstrução anatômica nas vias lacrimais.

Para análise dos resultados, foi aplicado o teste de MannWhitney, para comparação das idades em anos no momento da realização da irrigação das vias lacrimais e o teste do Quiquadrado, para identificar possíveis relações entre sexo, raça, uni ou bilateralidade dos sintomas e incidência de obstrução anatômica das vias lacrimais entre os grupos. Fixou-se em 0,05 ou 5\% ( $<<0,05)$ o valor de rejeição da hipótese de nulidade.

\section{RESULTADOS}

Os dois grupos são semelhantes quanto à idade em anos, sexo e raça; com respectivos resultados estatísticos: $\mathrm{p}=0,07$; $\mathrm{p}=0,63 ; \mathrm{p}=0,68$.

Sintomas bilaterais de epífora e/ou secreção ocular constante foram encontrados em 25 pacientes $(83,33 \%)$ do grupo A e em 335 pacientes $(50,38 \%)$ do grupo $\mathrm{B}(\mathrm{p}=0,0008)$ (Gráfico 1$)$.

A irrigação das vias lacrimais foi realizada em ambos os olhos, mas consideramos como dados do trabalho o resultado da irrigação apenas nos olhos com epífora e/ou secreção ocular constante. Foram estudados então 1.056 olhos, sendo 55 do grupo A e 1.001 do grupo B.

A obstrução anatômica das vias lacrimais foi encontrada em 18 olhos do grupo A $(32,73 \%)$ e 822 olhos do grupo B $(82,11 \%)(\mathrm{p}<0,0001)$ (Gráfico 2).

A agenesia ou imperfuração do ponto lacrimal foi encontrada em 34 pacientes do grupo B e em nenhum paciente do grupo A. Esses pacientes foram classificados como portadores de obstrução anatômica das vias lacrimais.

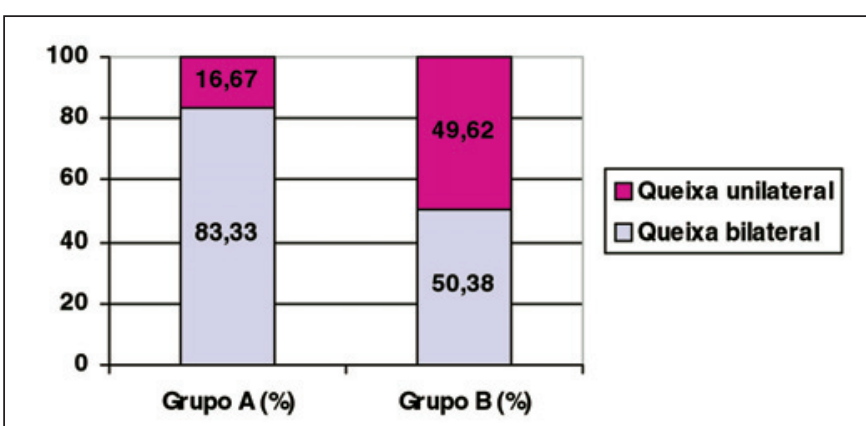

Grupo A: pacientes com síndrome de Down; Grupo B: pacientes controle. Fonte: Ambulatório de vias lacrimais do Departamento de Oftalmologia da Santa Casa de São Paulo no período de outubro de 1989 a julho de 2005.

Gráfico 1 - Porcentagem de queixa uni ou bilateral de epífora e/ou secreção ocular constante nos pacientes dos grupos A e B 


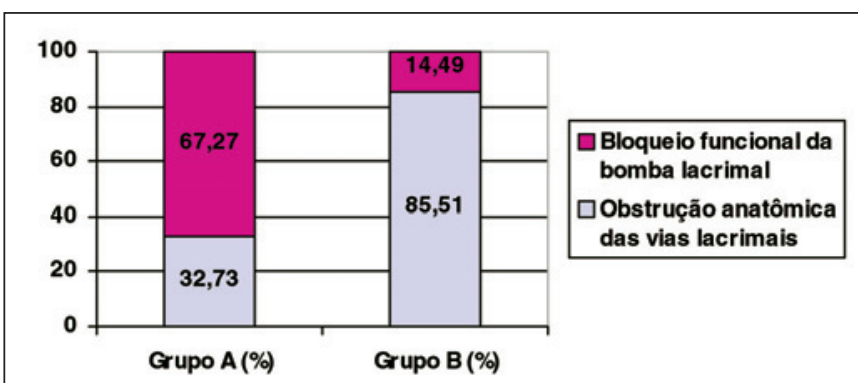

Grupo A: pacientes com síndrome de Down;Grupo B: pacientes controle. Fonte: Ambulatório de vias lacrimais do Departamento de Oftalmologia da Santa Casa de São Paulo no período de outubro de 1989 a julho de 2005.

Gráfico 2 - Resultado da prova de irrigação das vias lacrimais nos olhos com epífora e/ou secreção ocular constante dos pacientes dos grupos A e B

\section{DISCUSSÃO}

O número de portadores da síndrome de Down vem aumentando sensivelmente, devendo-se possivelmente ao maior número de gestações em mulheres acima de 35 anos e ao aumento da longevidade desses pacientes ${ }^{(7-8,10)}$.

$\mathrm{O}$ avanço tecnológico da medicina tem propiciado condições para melhor avaliação e tratamento das alterações sistêmicas na síndrome de Down ${ }^{(15)}$. O aumento da longevidade se associa com maior atenção às outras manifestações da síndrome, na perspectiva de melhora da qualidade de vida desses pacientes ${ }^{(1)}$.

As alterações oculares são freqüentes nos pacientes com síndrome de Down. Dessa forma, o reconhecimento e o tratamento precoce dessas alterações contribuem sobremaneira para diminuir as várias dificuldades que essas crianças apresentam em sua integração social ${ }^{(1,5)}$.

Alguns autores encontraram prevalência de epicanto em $63 \%$ dos pacientes com síndrome de Down de 0 a 5 anos; $61 \%$ de 5 a 12 anos e $36 \%$ a partir dos 12 anos, dados semelhantes aos da literatura ${ }^{(5)}$. O epicanto é mais raramente encontrado a partir dos 10 anos de idade ${ }^{(14)}$.

Os pacientes com síndrome de Down apresentam distância interpupilar menor do que a população geral. Acredita-se que esse estreitamento esteja relacionado ao diâmetro craniano, que é menor nos pacientes com essa síndrome ${ }^{(3)}$.

Os testes estatísticos mostram que os grupos A e B são semelhantes quanto à idade $(\mathrm{p}=0,07)$, sexo $(\mathrm{p}=0,63)$ e raça $(\mathrm{p}=0,68)$ o que permite a comparação dos resultados da prova de irrigação das vias lacrimais entre os dois grupos.

Dos 695 pacientes do nosso trabalho, 4,32\% apresentavam o diagnóstico de síndrome de Down com queixas de epífora. Isso reflete uma incidência maior do que a relatada na literatura, mas pode ser justificada por ser a Santa Casa de São Paulo um hospital de referência nas diversas especialidades.

A média de idade dos pacientes, ao realizar a prova de irrigação das vias lacrimais, foi maior no grupo A. Isso se deve, possivelmente, à presença das alterações sistêmicas encontradas na síndrome de Down, que posterga a procura ao oftalmologista e também dificulta a liberação clínica para o procedimento anestésico.

A análise comparativa da incidência de obstrução anatômica das vias lacrimais mostrou diferença estatisticamente significante entre os dois grupos $(\mathrm{p}<0,0001)$. Observamos, então, que apenas $32,73 \%$ dos olhos com epífora congênita do grupo A, tinham obstrução anatômica das vias lacrimais, enquanto no grupo B, isso ocorria em $82,11 \%$ dos olhos estudados.

A maioria dos pacientes do grupo A $(83,33 \%)$ apresentavam queixa bilateral, enquanto no grupo B apenas 50,38\% o faziam. Essa diferença também foi estatisticamente significante $(\mathrm{p}=0,0008)$.

As alterações do posicionamento palpebral, como epicanto, rima palpebral oblíqua, estreitamento da distância interpupilar e hipotonia da musculatura palpebral são as possíveis causas para a disfunção da bomba lacrimal em pacientes com síndrome de Down. A maior freqüência de queixa bilaterais nesses pacientes reforça a origem sistêmica dessa disfunção. São necessários, novos trabalhos, com a realização de dacriocistografia, dacriocintilografia, para confirmação dessas hipóteses.

\section{CONCLUSÕES}

Conclui-se que a maioria das causas de epífora congênita em pacientes com síndrome de Down é decorrente do bloqueio funcional da bomba lacrimal, diferente do que ocorre em pacientes sem a síndrome, em que a principal causa é a obstrução anatômica das vias lacrimais. Acreditamos que a propedêutica das vias lacrimais deva ser realizada nos pacientes com síndrome de Down, quando houver queixas, pois a obstrução anatômica pode estar presente, necessitando de diagnóstico e tratamento precoce.

\section{ABstraCT}

Purpose: To describe the causes of congenital epiphora in patients with Down syndrome. Methods: Retrospective study of 695 patients with congenital epiphora, of the Lacrimal Sector of the Department of Ophthalmology, São Paulo "Santa Casa", Brazil, between October 1998 and July 2005. This study analyzed: the main symptom of continuous epiphora or mucous discharge, which affected one or both eyes, since birth. Subjects were separated in to two groups: group A, with 30 patients with Down syndrome and group B, with 665 control patients. The lacrimal evaluation was performed by the throw irrigation test after general anesthesia. Results: Both groups were statistically similar regarding age $(p=0.07)$, sex $(p=0.63)$ and race $(\mathrm{p}=0.68)$. Bilateral symptoms were more frequent in group A ( $\mathrm{p}=0.0008)$. Anatomic obstruction of the lacrimal canal was present in $32.73 \%$ of group A and in $85.51 \%$ of group $\mathrm{B}(\mathrm{p}<0.0001)$. Conclusions: The most frequent cause of congenital epiphora in Down syndrome is due to functional block of the lacrimal pump. Eyelid muscle hypotony and eyelid changes, common in Down syndrome, is the main hypothesis 
of lacrimal dysfunction, but further studies will be necessary for confirmation.

Keywords: Lacrimal apparatus diseases/congenital; Lacrimal duct obstruction/etiology; Down syndrome/complications; Eye diseases

\section{REFERÊNCIAS}

1. Matayoshi S, Sardinha M, Cozac AL, Araf D, Moura EM. Síndrome de Down e alterações de vias lacrimais. Arq Bras Oftalmol. 2003;66(4):481-4.

2. Nussabaum RL, Mcinnes RR. Thompson \& Thompson: Genética Médica. 6a ed. Rio de Janeiro; Guanabara Koogan; 2002.

3. Jaeger EA. Ocular findings in Down's syndrome. Trans Am Ophthalmol Soc. 1980;798:808-45.

4. Smith DV. Síndromes de mal formações congênitas. 3a ed. São Paulo, Manole; 1985.

5. Cunha RNP, Moreira JBC. Manifestações oculares em crianças e adolescentes com Síndrome de Down. Arq Bras Oftalmol. 1995;58(3):152-7.
6. Hall B. Mongolism in newborn infants. An examination of the criteria for recognition and some speculations on the pathogenic activity of the chromosomal abnormality. Clin Pediatr (Phila). 1966;5(1):4-12.

7. Berk AT, Saatci AO, Ercal MD, Tunc M, Ergin M. Ocular findings in 55 patients with Down's syndrome. Ophthalmic Genet. 1996;17(1):15-9.

8. Kim JH, Hwang JM, Kim HJ, Yu YS. Characteristic ocular findings in Asian children with Down's Syndrome. Eye. 1996;17(1):15-9.

9. Soares EJC, França VP. Sistema lacrimal de drenagem. Rio de Janeiro: Cultura Médica; 1999. p.10-6.

10. Shapiro MB, France TD. The ocular features of Down's syndrome. Am J Ophthalmol. 1985;99(6):659-63.

11. Catalano RA. Down Syndrome. Survey Ophthalmol. 1990;34(5):385-98.

12. Kanski JJ. Oftalmologia clínica: uma abordagem sistêmica. 4a ed. Rio de Janeiro: Rio Med Livros; 2004.

13. Coats DK, McCreery KM, Plager DA, Bohra L, Kim DS, Paysse EA. Nasolacrimal outflow drainage anomalies in Down's syndrome. Ophthalmology. 2003;110(7):1437-41.

14. Solomons G, Zellweger H, Jahnke PG. Four commom eye signs in Mongolism. Am J Dis Child. 1965;110:46-50.

15. Baird PA, Sadovnick AD. Life expectancy in Down syndrome. J Pediatr. 1987;110(6):849-54.

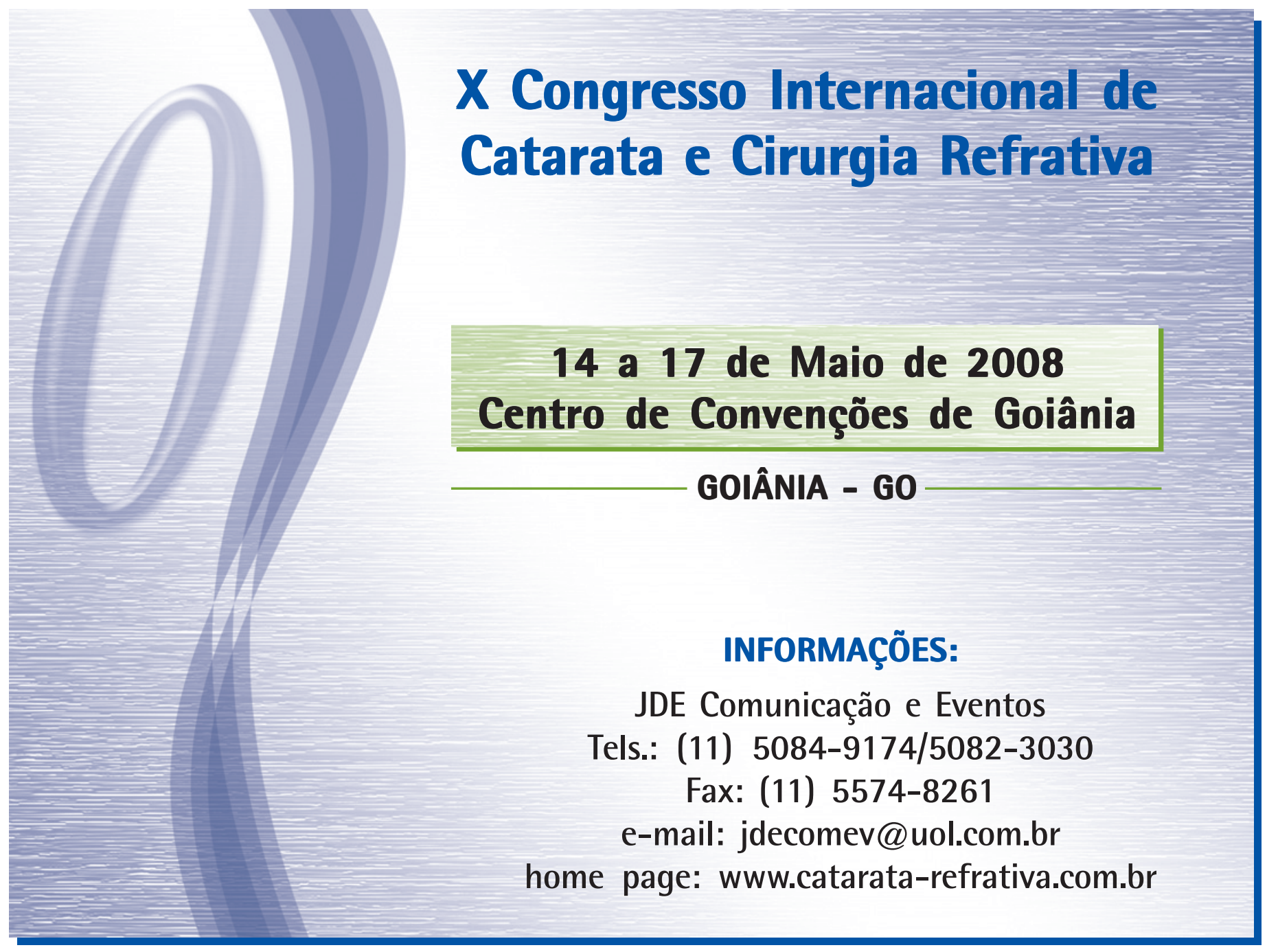

